\title{
Strategies for the Connection of Distributed Power Generation Units to Distorted Networks
}

\author{
Cristian Blanco, David Reigosa, Fernando Briz and Juan M. Guerrero \\ University of Oviedo. Dept. of Elect., Computer \& System Engineering. Gijón, 33204, Spain \\ blancocristian@uniovi.es,diazdavid@uniovi.es, fernando@isa.uniovi.es, guerrero@isa.uniovi.es
}

\begin{abstract}
Connection of Distributed Power Generators (DPGs) to a utility grid requires the synchronization between the DPG and the grid voltages with the goal of controlling the power flow and limiting the currents at instant that the switch or breaker closes. Synchronization methods typically match the positive sequence voltage component of the two systems being connected, the connection being realized when the error is smaller than a pre-set threshold. While synchronization using the positive sequence voltage provides adequate results in the case of undistorted grid voltages, it can be inadequate if the grid is polluted, e.g. in case of weak grids. Instantaneous voltage mismatches between the DPG and the grid, e.g. due to the harmonic content or unbalances in the grid and/or DPG voltages..., at the instant that the breaker closes, can cause overcurrents and result in unwanted protection tripping or equipment damage, even if the corresponding positive sequence voltages perfectly match.

This paper analyzes the synchronization and connection of DPGs to highly distorted grids with the goal of limiting the overcurrent due to voltage mismatch. Once the connection is realized, a transition to the conventional positive sequence voltage based synchronization occurs, as this is adequate to control the power flow in steady state.
\end{abstract}

Index Terms-microgrids, power system interconnection, power system harmonics, phase locked loops, current control

\section{INTRODUCTION}

I $\mathrm{T}$ is widely accepted that one of the priorities for the following decades is to increase the use of renewable energy. The introduction of "clean generation units" has boosted the evolution from the traditional centralized generation model to a distributed generation model. Centralized generation is a mature technology with good economies of scale, coal, gas, oil, nuclear and hydropower being the primary resources. However, energy needs to be routed through long distances, what increases the transmission losses. Distributed generation (DG) based both on renewable and no renewable energy resources has been traditionally used at small scale in rural areas with limited access to the main grid. Benefits on DG include reduction of size, number and level of saturation of transmission lines as well as reduction of the transmission losses. Massive utilization of DG places however several challenges due to the variability of renewable energy resources, including stability and dispatchability issues and the absence of a proper market design.

This work was supported in part by the Research, Technological Development and Innovation Programs of the Ministry of Science and Innovation under grant MICINN-10-CSD2009-00046 and by the Personnel Research Training Program funded by the Regional Ministry of Education and Science of the Principality of Asturias under grant BP11-107
DPG units are often connected to form a microgrid, which can operate as an island or connected to the utility grid [1]. In the island mode, a DPG unit (master unit) is responsible of setting the magnitude and frequency of the supplied voltage at the point of common coupling (PCC) [1], the remaining DPGs (slaves) automatically synchronize with the PCC voltage to inject power on demand. In the grid-connected mode, the voltage magnitude and phase are set by the grid, the DPGs units typically working as power sources.

Synchronization, i.e. the measurement of the grid/microgrid voltage magnitude, frequency and phase angle, by all the units injecting power, will be key go guarantee a robust and stable operation of the system and adequate power quality. Synchronization methods typically try to isolate the positive sequence component of the fundamental voltage, often implementing mechanisms for the compensation of disturbances like harmonics and voltage unbalances for this purpose [2]. Especially critical is the instant when the physical connection between a DPG unit and the microgrid/grid is realized. Having the same voltage in both sides of the breaker is needed to avoid overcurrents that may damage the DPG or tripping the protections. This is not guaranteed in distorted grids when the synchronization is realized using the positive sequence voltage only, which can be of especial importance in weak grids, e.g. those located at the end of long distance transmission lines for centralized power distribution scenarios or near low-power DPG units in distributed power generation scenarios.

In this paper, the synchronization and connection of DPGs to highly distorted grids is analyzed, with the goal of reducing the induced overcurrents due to the instantaneous voltage mismatch. In the proposed method, both the positive sequence as well as the harmonic components of the grid voltage are considered for the synchronization with the goal of reducing the voltage mismatch at the instant that the breaker closes. Once the connection process is finished, the disturbances are gradually removed from the reference used for the synchronization, the positive sequence component of the voltage being eventually used in order to guarantee precise control the active and reactive power flows.

The paper is organized as follows: section II reviews the control strategies for islanded and grid connected DPG units. Section III presents the proposed method, simulation and experimental results to validate it are given in Section IV and $\mathrm{V}$ respectively. Finally, Section VI presents the conclusions. 


\section{CONNECTION OF DPGS TO A DISTORTED UTILITY GRID USING THE FUNDAMENTAL VOLTAGE}

Fig. 1 shows a generic control block diagram of a voltage source converter working in Voltage Control Mode (VSCVCM). It consists of an inner current loop (block in red) that controls the inverter output current $\left(R e g_{i}\right)$ and an outer voltage loop (block in green) that controls the output filter voltage

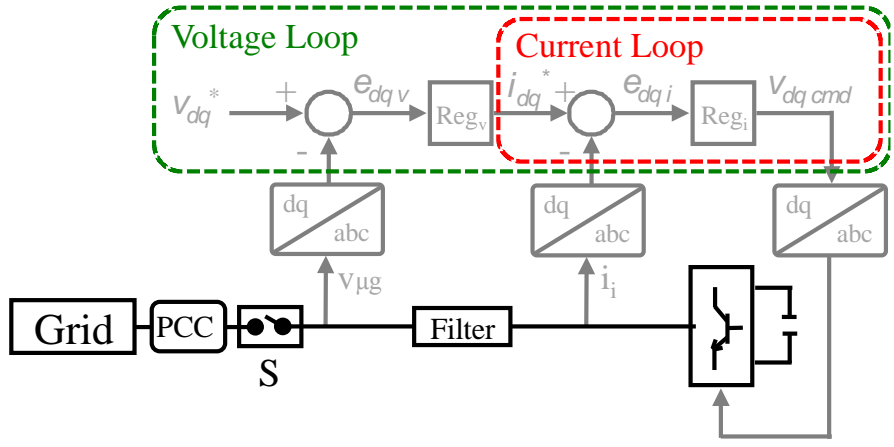

Fig. 1.- Generic VSC control diagram with voltage and current control loops.

The three phase voltages and currents are transformed from $a b c$ to $d q$ quantities in a reference frame synchronous with the grid voltage fundamental component using (1)-(2), where 0 and $\quad 0$ are the phase angle and frequency of the fundamental component of the grid voltage. Synchronous PI regulators $\left(\operatorname{Reg}_{i}\right.$ and $\operatorname{Reg}_{v}$ in Fig. 1) are normally used to control the fundamental components of the voltage and current respectively.

$$
\begin{aligned}
f_{d q} & =2 / 3\left(f_{a}+f_{b} e^{* 2^{*} / 3}+f_{c} e^{* * 4^{*} / 3}\right) e^{j^{*} 0} \\
& ={ }_{0} d t
\end{aligned}
$$

The voltage reference, $v_{d q}{ }^{*}$ (see Fig. 1), comes from a synchronization algorithm, typically based on a phase-lockedloop (PLL) [2]. To provide a clean estimation of the positive sequence component of the fundamental voltage, PLL algorithms often implement mechanisms to compensate or eliminate unbalances or harmonics that typically exists in the grid voltage.

Fig. 2 shows the $i_{d q g}$ current when a connection to a distorted grid is performed using a clean estimation of the fundamental component of the PCC. It is observed that the voltage mismatch between the breaker terminals produces a

a)

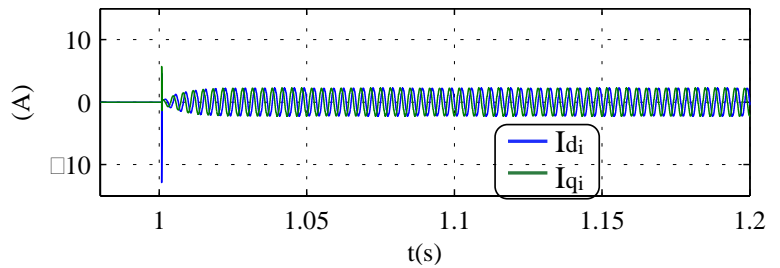

b)

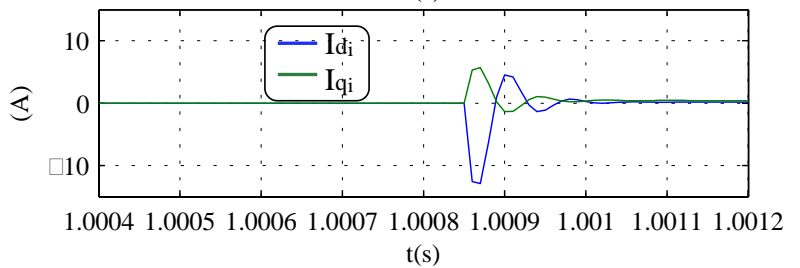

Fig. 2.- Connection of a DPG to a distorted grid a) grid current $i_{d q g}$, b) zoom of $i_{d q g}$ at the instant that the breaker closes. overcurrent after the breaker is closed, its magnitude being a function of the voltage difference between the DPG and the grid, the DPG output filter and the grid impedance. LC and LCL filters are the most common choice for the DPG output filter, the transient current after the connection for both filter designs is analyzed following.

\section{A. Connection of VSCs to distorted grids through LC filters}

Fig. 3 shows the equivalent circuit of a three-phase VSC connected to the utility grid through an LC filter. The transmission line and inductance filter are modeled by an inductance $\left(\mathrm{L}_{\mathrm{TL}}\right.$ and $\mathrm{L}_{\mathrm{i}}$ ) and a resistance $\left(\mathrm{R}_{\mathrm{TL}}\right.$ and $\mathrm{R}_{\mathrm{i}}$ ) respectively. The capacitor filter is modeled by a capacitance $C$ with a series resistance, $R_{c}$.

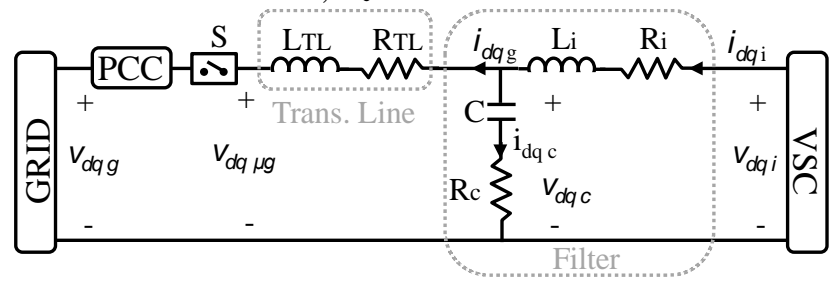

Fig. 3- Equivalent circuit of a VSC connected to a grid using an LC filter.

During the synchronization process and before the breaker is closed, the microgrid voltage $v_{d q u g}$ is normally made equal to the positive sequence component of the grid fundamental voltage. This means that if the grid voltage contains only a fundamental positive sequence component, no current will flow once the breaker is closed, as no differential voltage between the VSC and grid voltage exists. On the contrary, a voltage mismatch between the grid and the VSC output voltages (i.e. at the breaker " $S$ " terminals, see Fig. 1) will exist if the grid voltage is distorted, which will result in an overcurrent once the breaker is closed.

In the following discussion, it is assumed that the grid voltage consists of the fundamental voltage component and a single harmonic component voltage of order $h$ and magnitude $\left|v_{d q g h}\right|$. It can also be realistically assumed that the capacitor harmonic current equals the grid harmonic current $\left(i_{d q g h}=i_{d q c h}\right)$, since the reference of the VSC current regulator only contains the fundamental component and the inverter inductance removes the produced high frequency overcurrent.

The transfer function between the grid harmonic current, $i_{d q g h}$, and the grid harmonic voltage, $v_{d q g h}$, is given by (3), where $L$ is the transmission line inductance $\left(L=L_{L T}\right), R$ is the equivalent resistance of the grid and the filter capacitor $\left(R=R_{T L}+R_{c}\right)$ and $C$ is the filter capacitor. It can be safely assumed that $R^{2} / L^{2}<<4 /(L C)$, therefore (3) corresponds to underdamped system, whose poles are (4). The differential voltage across the breaker terminals in a reference frame synchronous with the grid frequency is (7), the induced current being (8). Note that a harmonic component at a frequency $\omega_{g h}=h \omega_{0}$ transforms into a harmonic component at a frequency $\omega_{g h}-\omega_{o}$ in a $d q$ reference frame synchronous with the fundamental excitation. The induced current is seen to consist of a transient term and a steady state term. The time constant of the transient term is (5) and its resonance frequency (6). 
The steady state term has the same frequency as the input signal and its magnitude depends on the filter and transmission line parameters (9) and (10).

$$
\begin{aligned}
& \frac{i_{\text {dqgh }}}{v_{\text {dqgh }}}=\frac{\frac{s}{L}}{s^{2}+s \frac{R}{L}+\frac{1}{L C}} \\
& p_{1}=+j \times_{r} ; p_{2}=j \times_{r} ; \\
& =\frac{R}{2 L} \\
& r=\sqrt{\frac{1}{C L} \frac{R^{2}}{4 L^{2}}} \\
& \left.V_{\text {dqgh }}=\left|V_{\text {dqgh }}\right| \theta^{i *} \text { gh } \quad 0\right) * t \\
& \left.i_{\text {dqgh }}=i_{\text {steady }}+i_{\text {trans }}=\left|i_{\text {steady }}\right| e^{j\left(\begin{array}{ll}
\text { gh } & 0
\end{array}\right) t+}\right)+\frac{\left|V_{\text {dqgh }}\right|}{L_{r}} e^{t} e^{j} r t \\
& \left|i_{\text {steady }}\right|=\frac{\left|v_{\text {dgoh }}\right|}{\sqrt{R^{2}+\left({ }_{g h} L \frac{1}{{ }_{g h} C}\right)^{2}}} \\
& =\tan 1\left(\frac{{ }_{g h} L \frac{1}{{ }_{g h} C}}{R}\right)
\end{aligned}
$$

\section{B. Connection of VSCs to distorted grids through LCL filters}

Fig. 4 shows the equivalent circuit of a VSC using an LCL filter. The transmission line is connected between the LCL filter output and the breaker $S$, being modeled as in the previous subsection. The inverter is synchronized with the positive sequence component of the fundamental voltage at the PCC, $i_{d q i}$ and $v_{d q u g}$ only consisting therefore of the fundamental component.

When the breaker closes, the current to voltage transfer function is also modeled by (3), the equivalent inductance resulting from the series connection of the line inductance and the LCL filter grid-side inductance $\left(\mathrm{L}=\mathrm{L}_{\mathrm{TL}}+\mathrm{L}_{\mathrm{g}}\right)$ and the equivalent resistance $R$ being the result of the series connection of the transmission line, LCL filter grid-side inductance resistance and filter capacitor resistance, i.e. $R=R_{T L}+R_{g}+R_{c}$.

The time response for this case can also be described by (8), and is seem to consist of a transient term with a time constant (5) and resonance frequency (6), and a steady state term with the same frequency as the PCC harmonic voltage $\left(\omega_{g h}-\omega_{o}\right)$, of magnitude and phase (9) and (10) respectively.

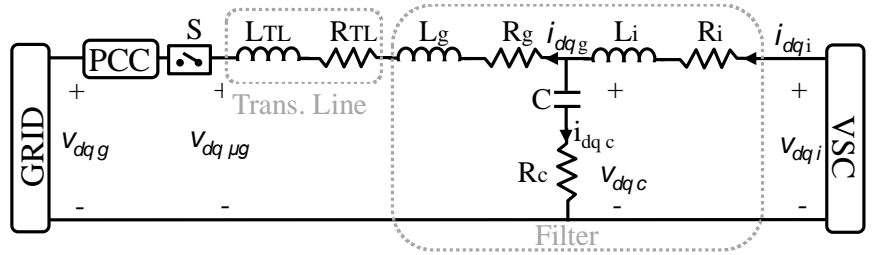

Fig. 4.- Equivalent circuit of a VSC connected to a grid using an LCL filter

\section{CONNECTION OF DPGS TO A DISTORTED UTILITY GRID USING FUNDAMENTAL AND HARMONIC VOLTAGES}

To limit the transient overcurrents following the connection between the DGs and the grid, both the fundamental and the harmonic voltage components can be considered for the synchronization strategy.

Fig. 5 shows control block diagram of the proposed strategy. A combination of proportional-integral (PI), (11) and resonant (RES) regulators, (12), in $d q$ reference frame synchronous with the grid fundamental frequency are used (13), both for the current and voltage. The PI synchronous regulators (11) are used to control the fundamental voltage and current components, while each RES controller (12) is used to control a specific harmonic component of the voltage and current, with $n$ being the harmonic order. It is noted that in a harmonic of order $h$ in the stationary reference frame results in a harmonic of order $n=h-1$ in a reference frame synchronous with the fundamental component of the grid voltage,

$$
\begin{aligned}
& P I(s)=K_{p}+\frac{K_{i}}{s} \\
& R E S_{n}(s)=\frac{K_{P_{\text {RES } n}} \times s^{2}+K_{p_{\text {RES } n}} 2 \times C \times s+\left(n \times \times_{0}\right)^{2}+C^{2}}{s^{2}+\left(n \times{ }_{0}\right)^{2}}
\end{aligned}
$$

$\operatorname{PI}-\operatorname{RES}(s)=\operatorname{Pl}(s)+R E S_{n}(s)$

The voltage reference $v_{d q}{ }^{*}$ and both the measured voltage and current $\left(v_{d q \mu g}\right.$ and $\left.i_{d q i}\right)$ (see Fig. 5) are not filtered (i.e. harmonics are not removed). The PLL described in [2] was used for the synchronization, as it provides disturbance rejection capability. The strategy to synchronize and connect the VSC to a distorted grid is as follows:

1. $\underline{S W}_{1}=\mathrm{ON}, \mathrm{SW}_{2}{ }_{2}=\mathrm{ON}$, Breaker $S=$ open. The PI-RES voltage controller provides the required current command $i_{d q}{ }^{*}$ (Fig. 5) to the PI-RES current controller to force the same voltage at both sides of the breaker terminals (fundamental and harmonics).

2. $\underline{S W}_{1}^{*}=\mathrm{OFF}, \mathrm{SW}_{2}=\mathrm{ON}$, Breaker $S=$ closed. Once the voltage error is smaller than a certain threshold, the breaker is closed. Following this, the input of the voltage controller is made equal to zero using $\mathrm{SW}_{1}{ }_{1}$ (see Fig. 5). By doing this, the voltage loop is disabled, the PI-RES output being locked to the instantaneous value that it has before the breaker closed.

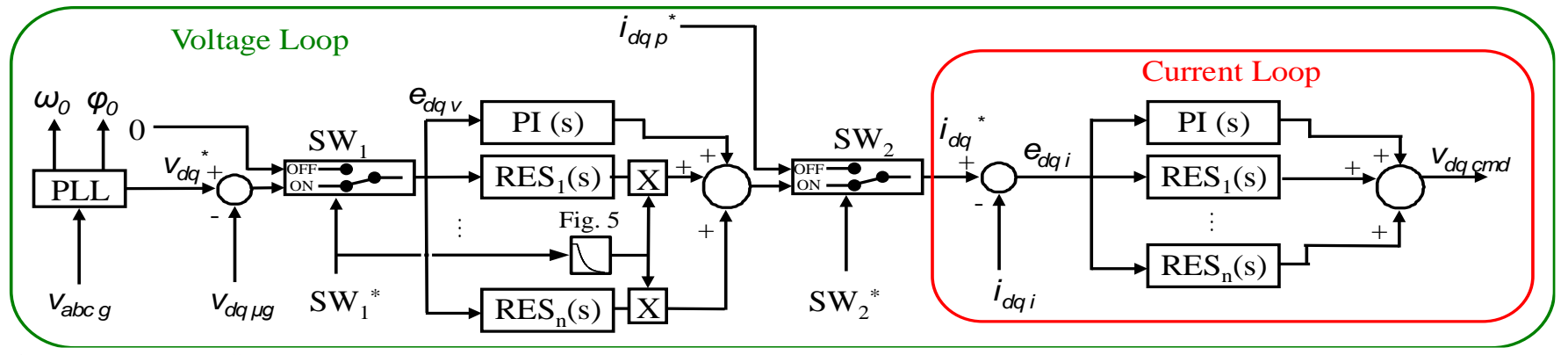

Fig. 5.- PI-RES controller structure. 
At the same time that $\mathrm{SW}^{*}{ }_{1}$ deactivates the voltage control loop, the resonant voltage controller output is gradually eliminated by multiplying the RES output (12) by a weighting factor of the form shown in Fig. 6. By doing this, the VSC gradually decreases the amount of harmonics injected, eventually only the fundamental current being injected.

3. $\underline{S W}_{1}{ }_{1}=\mathrm{OFF}, \mathrm{SW}_{2}{ }_{2}=\mathrm{OFF}$, Breaker=closed: Once the current harmonics are removed, the input to the current regulator is made equal to the current command $i_{d q p}{ }^{*}$ coming e.g. a power control loop, Switch $\mathrm{SW}_{2}{ }_{2}$ being used for this purpose.

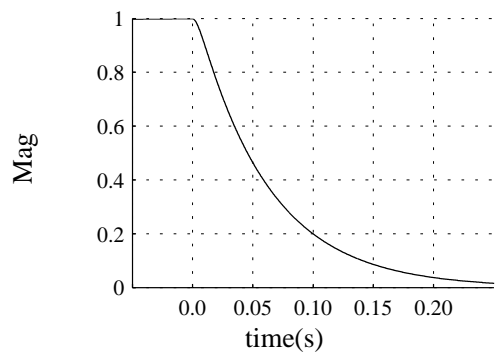

Fig. 6 - Harmonic current adaptive gain.

\section{Simulation RESUlts}

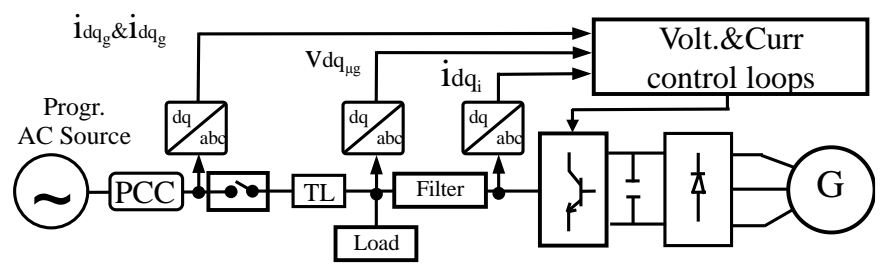

Fig. 7.- Proposed simulation setup.
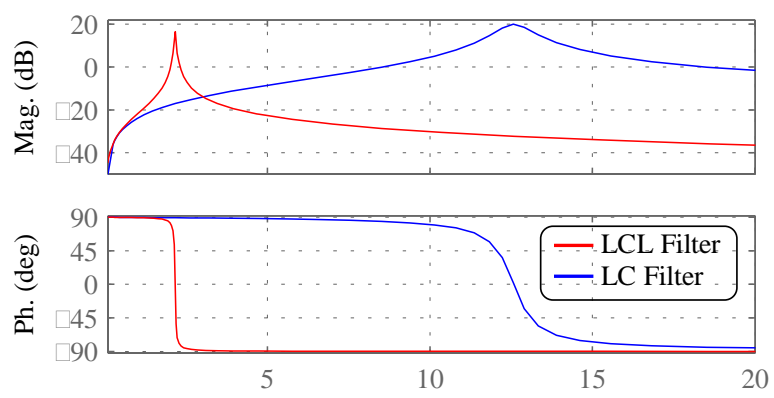

Frequency $(\mathrm{kHz})$

Fig. 8.- a) Magnitude and b) phase of the frequency response function of $i_{g h} / v_{g h}$ for the LC (blue) and LCL (red) filters.

The proposed method has been tested by simulation, the scenario and the configuration parameters being shown in Fig. 7 and Table I respectively. A programmable AC source was used to emulate the grid voltage, the grid voltage distortion consisting of a $-5^{\text {th }}$ harmonic with a magnitude of $2.6 \%$, which is below the limit of $4 \%$ allowed by the grid codes [4]-[6]. A short transmission line (TL, see Fig. 7) has been used to emulate the DPG connection to the PCC. Fig. 8 shows the frequency response of $i_{\text {dqgh }} / v_{\text {dqgh }}$ transfer function (3), using LC (blue) and LCL (red) filters.
TABLE I

SIMULATION AND EXPERIMENTAL PARAMETERS

\begin{tabular}{lll}
\hline \hline Symbol & \multicolumn{1}{c}{ Description } & \multicolumn{1}{c}{ Value } \\
\hline $\mathrm{L}_{\mathrm{i}}$ & Inverter side filter inductance & $2.196 \mathrm{mH}$ \\
$R_{i}$ & Inverter side filter resistance & $0.05 \Omega$ \\
$C$ & Capacitor Filter & $10 \mu \mathrm{F}$ \\
$R_{c}$ & Capacitor ESR & $0.0536 \Omega$ \\
$\mathrm{L}_{\mathrm{g}}$ & Grid side filter inductance & $0.5 \mathrm{mH}$ \\
$R_{g}$ & Grid side filter resistance & $0.05 \Omega$ \\
$L_{T L}$ & Transmission Line inductance & $0.02 \mathrm{mH}$ \\
$R_{T L}$ & Transmission Line impedance & $0.1 \Omega$ \\
& Positive sequence voltage magnitude & $380 \mathrm{~V}$ \\
& (rms) & \\
& -5 & $10 \mathrm{~V}$ \\
$K_{p} \mathrm{~V}$ & PI proportional constant & 0.1 \\
$K_{i} \mathrm{~V}$ & Voltage PI integral constant & 0.5 \\
$K_{p R E S} \mathrm{~V}$ & Voltage RES proportional constant & 1 \\
$\mathrm{C} \mathrm{V}$ & Voltage RES constant & 100 \\
$K_{p} \mathrm{C}$ & Current PI proportional constant & 8.4 \\
$K_{i} \mathrm{C}$ & Current PI integral constant & 60 \\
$K_{p} R E S \mathrm{C}$ & Current RES proportional constant & 1 \\
$\mathrm{C} \mathrm{C}$ & Current RES constant & 300 \\
\hline \hline & &
\end{tabular}

a)

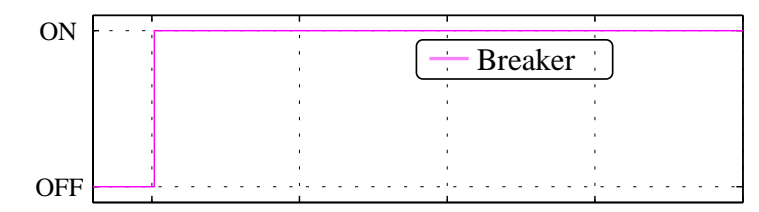

b)

c)

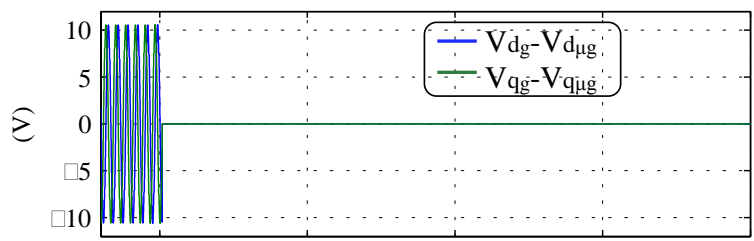

d)
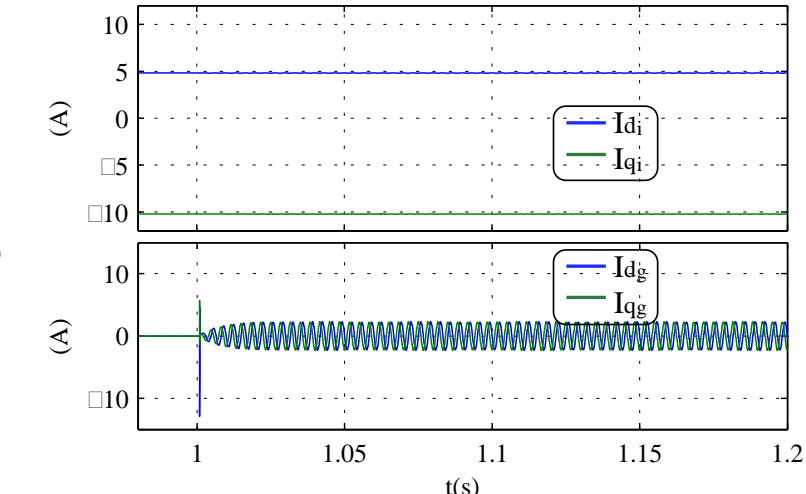

e)

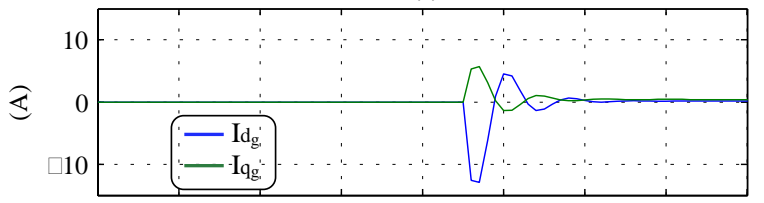

$1.00041 .00051 .00061 .00071 .00081 .00091 .001 \quad 1.00111 .0012$

$\mathrm{t}(\mathrm{s})$

Fig. 9.- Simulation results. DPG with LC filter synchronization using the positive sequence component of the fundamental voltage. a) Breaker control signal, b) $d q$ components of the differential voltage at the breaker terminals, c) inverter current $i_{d q i}$ d) grid current $i_{d q g}$, e) zoom of $i_{d q g}$ at the instant that the breaker closes. 
a)

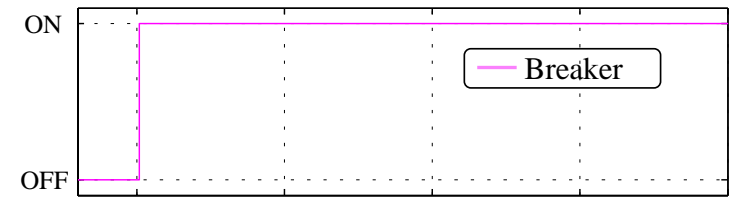

b)

c)

d)

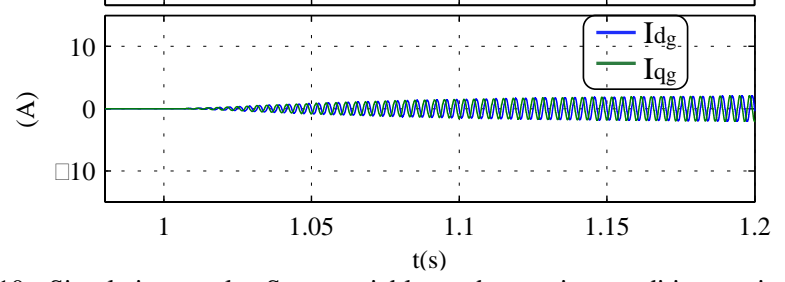

Fig. 10.- Simulation results. Same variables and operating conditions as in Fig. 9 using the proposed method.

The resulting resonance frequencies (6) are $12.5 \mathrm{kHz}$ for the LC filter and $2.25 \mathrm{kHz}$ for the LCL filter respectively. It is noted that the LCL resonance frequency is typically lower than that of the LC filter since the resonance frequency decreases as the inductance that connects the capacitor filter and the PCC increases.

Following, synchronization using the positive sequence component of the fundamental voltage described in Section II and the method proposed and described in Section III are compared, both for the case of a LC filter and an LCL filter.

\section{A. Connection to a distorted utility grid using an LC filter}

Fig. 9 shows the simulation results when the positive sequence component of the fundamental voltage is used for synchronization for the case of an LC filter. Fig. 9a shows breaker state (Fig. 7), it is observed in the figure that it closes $\mathrm{t}=1 \mathrm{~s}$. The $d q$ components of the differential voltage between both sides of the breaker terminals (voltage error) are shown in Fig. 9b, while the $d q$ components of the current circulating through the inverter ( $i_{d q_{i}}$ in Fig. 7) are shown in Fig. 9c. Fig. $9 \mathrm{~d}$ show the current that circulates between the filter output and the grid, Fig. 9e shows the detail of this current at the instant the breaker closes.

It is observed from Fig. 9d that the differential voltage shown in Fig. 9b produces a transient grid current, of peak value of $\approx 14 \mathrm{~A}$, which is several times bigger than the steady state current, its dynamics being given by (8)-(10). The dumped resonance frequency (see Fig. $9 \mathrm{e}$ ) is $\approx 12.5 \mathrm{kHz}$, which is in accordance with (6). A current harmonic at 300 $\mathrm{Hz}$ (-5th harmonic) exists in steady state (see Fig. 9d), its magnitude being given by (9). a)

b)

c)

d)

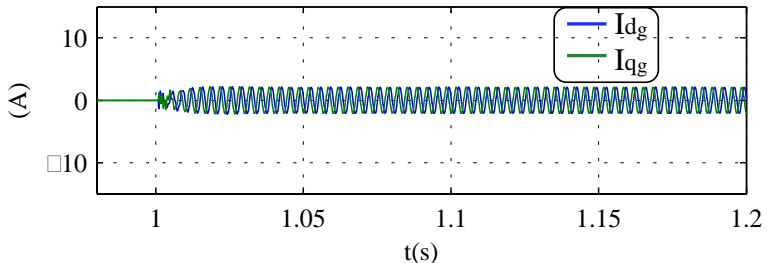

e)

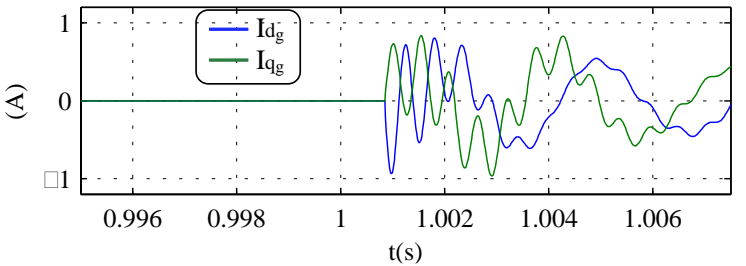

Fig. 11.- Simulation results. Same variables, operating conditions and synchronization strategy as in Fig. 9, using an LCL filter.

Fig. 10 shows the same simulation results as in Fig. 9 for the proposed method described in Section III. It is observed from Fig. 10b that the differential voltage at the breaker terminals is zero, as both fundamental and harmonic components are considered during the synchronization process. It is observed that with the proposed method, the grid overcurrent in Fig. 9d ( $i d q_{g}$ in Fig. 7) is almost completely cancelled (see Fig. 10d).

\section{B. Connection to a distorted utility grid using an LCL filter}

Fig. 11 shows the same simulation test as Fig. 9 but using an LCL filter. It is observed that the overcurrent at the instant that the breaker closes (see Fig. 11e) is smaller than for the LC case, since it is limited by the LCL filter grid-side inductance.

The resonance frequency of the dumped transient response agrees with the predicted one of $\approx 2.25 \mathrm{kHz}$ (see Fig. 8 and (6)), being lower than for the LC filter case (see Fig. 8). The higher the inductance between the filter capacitor and the PCC is, the smaller resonance frequency is. Similarly, the higher the inductance between the filter capacitor and the PCC is, the smaller is the transient time constant (5). It can be concluded that smaller connection inductances (transmission line plus filter inductances) results in larger transient overcurrents, but more damped. 
a)

b)

c)

d)

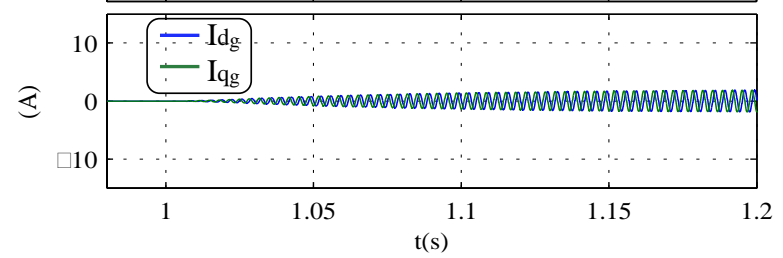

synchronization strategy as in Fig. 10, using an LCL filter.

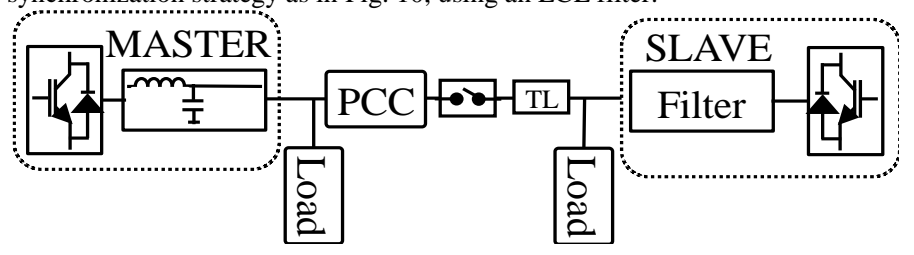

Fig. 13.- Experimental setup

Fig. 12 shows the same simulation results as Fig. 10 but for the case of the LCL filter. The same behavior for the inverter output current and the grid current (see Fig. 12c and 11d) as for the LC simulation test is observed.

It is concluded that the proposed method eliminates the transient overcurrent after the connection, for both LC and LCL filters.

\section{EXPERIMENTAL TESTS}

Fig. 13 shows the experimental setup. It consists of two VSCs (Master and Slave in Fig. 13). The master inverter is used to emulate the programmable AC source (see Fig. 7). It is a $50 \mathrm{~kW}$ inverter that feeds a local resistive load $(53 \Omega)$, it uses the same LC filter as in simulation (Table I). The slave inverter configuration is the same as in simulation (see Table I), and feeds a local resistive load (see Table I). The same tests used in simulation were repeated for the experimental verification.

\section{A. Connection to a distorted utility grid using an LC filter}

Fig. 14 shows the experimental results when the synchronization is realized using the positive sequence component of the fundamental voltage, for the case of an LC filter. a)

b)

c)

d)

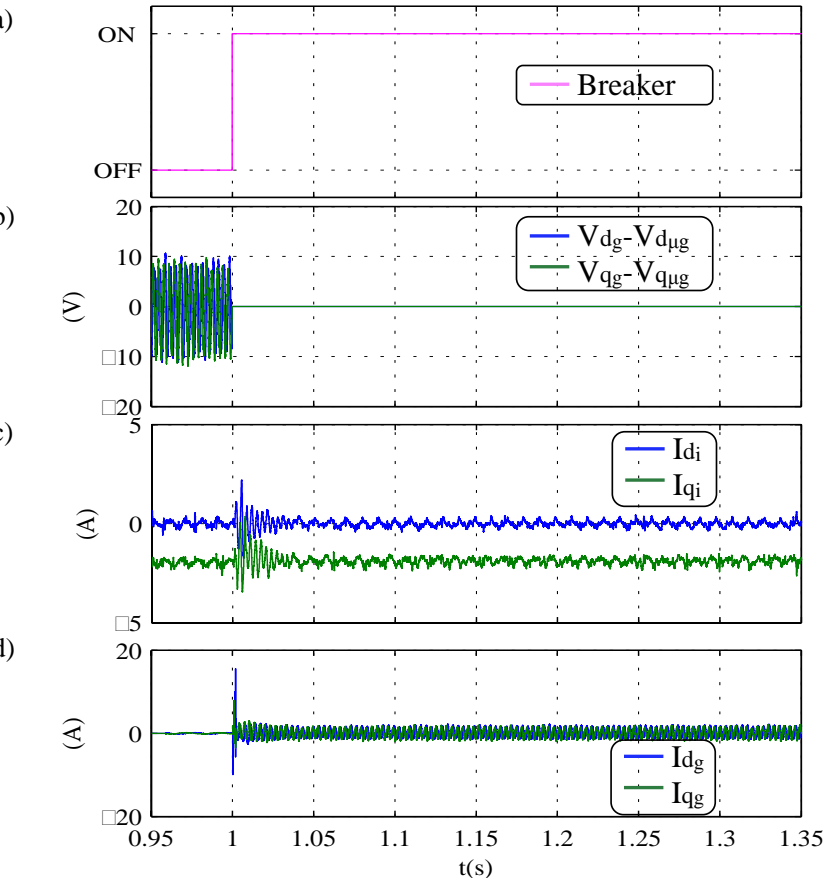

e)

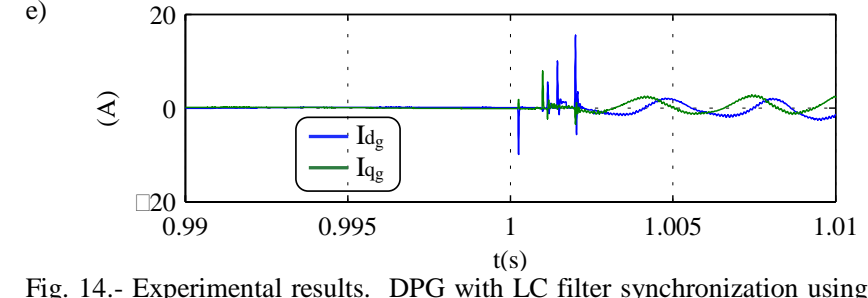

Fig. 14.- Experimental results. DPG with LC filter synchronization using the positive sequence component of the fundamental voltage. a) State of the breaker, b) $d q$ components of the differential voltage at the breaker terminals, c) inverter current $i_{d q i}$, d) grid current $i_{d q g}$, e) detail of $i_{d q g}$ at the instant that the breaker closes.

For $\mathrm{t}<1 \mathrm{~s}$ the slave inverter is synchronized to the fundamental component of the PCC voltage. The differential voltage at the breaker terminals is equal therefore to the PCC harmonic voltage components (see Fig. 14b), while the inverter current (see Fig. 14c) contains only the fundamental component.

At $\mathrm{t}=1 \mathrm{~s}$, the breaker closes (Fig. 14a). A transient increase of the grid current is observed (Fig. 14d). In addition, highorder harmonics appear at the inverter current (Fig. 14c, idq in Fig. 7) at the instant the breaker closes, which are compensated by the current regulator. The transient current is seen to have a resonance frequency of $\approx 14 \mathrm{kHz}$, with a peak current of $\approx 16 \mathrm{~A}$. It is noted that the current spikes observed in the $d q$ components of the grid current ( $i d q_{g}$ in Fig. 7) are caused by three-phase breaker rebounds (see Fig. 17d and 16e). The differences between the simulation and the experimental results would be due to differences in the line inductance (the cable mutual inductances have not been included in the simulations), which affect to the transient time constant (5), the resonance frequency (6) and peak current. 
a)

b)

c)

d)

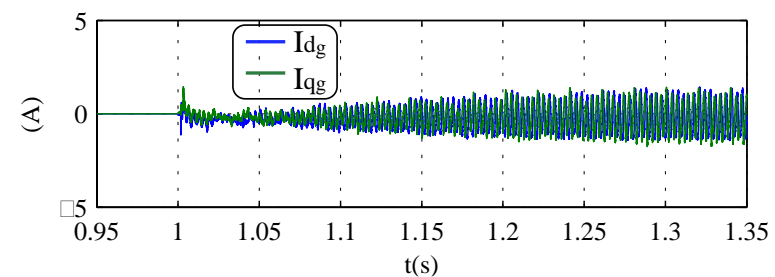

Fig. 15.- Experimental results. Same variables, operating conditions as in Fig. 14 , using the proposed method.

Fig. 15 shows the response of the proposed method. The slave VSC uses both the fundamental and the harmonic components of the voltage to feed the synchronization control loop, ideally providing zero differential voltage at the breaker terminals when it closes, (Fig. 15b). As for the simulation results, no overcurrent is observed when the breaker closes $(\mathrm{t}=1 \mathrm{~s}$, Fig. 15d). The injected current harmonics later reduce following the pattern shown in Fig. 6 (Fig. 15c), the grid current harmonics therefore increasing (see Fig. 15c and 14d). It is noted that once the high-order current harmonics are removed $(t>1.35 \mathrm{~s})$, the inverter only injects the fundamental component of the current.

\section{B. Connection to a distorted utility grid using an LCL filter}

Fig. 16 shows the experimental results when the synchronization is realized using the positive sequence component of the fundamental voltage, for the case of an LCL filter. As for the LC filter case, the high-order harmonics in the inverter current that appears at the instant the breaker closes, are compensated by the current regulator (see Fig. 16c). As for the simulation results (Fig. 11c), the peak current is smaller than for the case of the LC filter. The resonance frequency (Fig. 16d) is also smaller as for the LC filter case ( $\approx 2 \mathrm{kHz}$ ).

Good agreement among the simulation results (see Fig. 13) and the experimental results (see Fig. 16) is observed, differences being due to the line inductances, which have not been considered for the simulation analysis.

Finally, Fig. 17 shows the experimental results using the proposed method for the case of an LCL filter.

a)

c)

d)
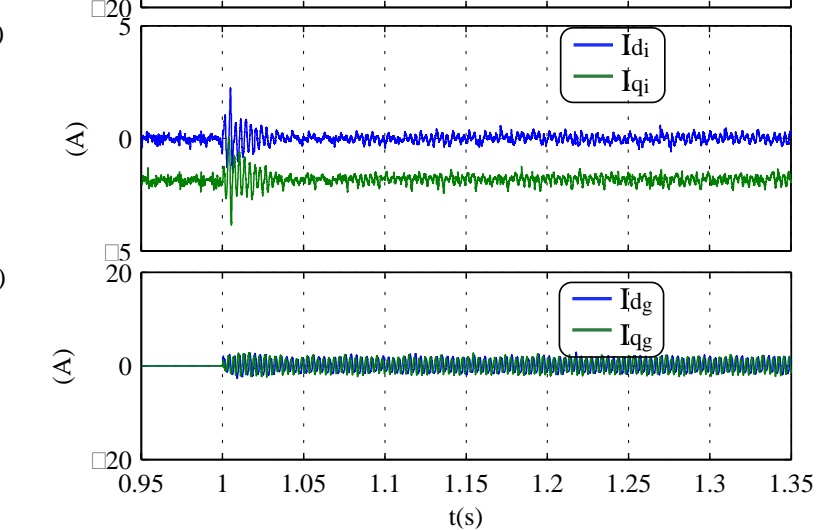

e)

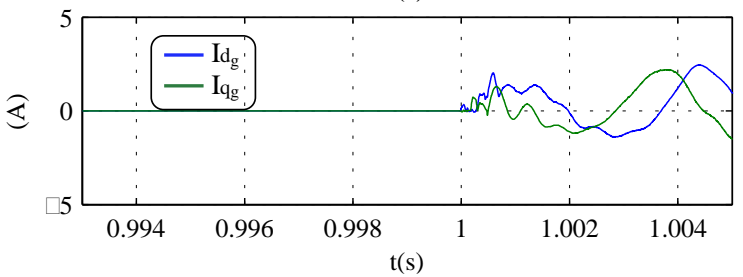

Fig. 16.- Experimental results. Same variables, operating conditions and synchronization method as in Fig. 14, using an LCL filter.

It is observed from Fig. 17b that the differential voltage at the breaker terminals at the instant it closes $(t<1)$ is negligible (it mainly consist of noise). It is observed in Fig. 17c that to match the grid voltage (fundamental+harmonic components), the inverter current contains both fundamental and harmonic components. No overcurrent is observed at the instant the breaker closes (Fig. 17d, $\mathrm{t}=1$ ), the inverter progressively decreasing later the amount of current harmonics being injected according to the pattern shown in Fig. 6 .

It is concluded that the proposed method provides a smooth connection as well as smooth transition to steady state, even for the case of highly distorted voltages at the PCC.

\section{CONCLUSIONS}

A method for the synchronization and smooth connection of DPGs to distorted networks has been proposed in this paper. The proposed method uses both the positive sequence component of the fundamental voltage as well as the grid voltage harmonics for the synchronization, avoiding transient overcurrents at the instant of connection of the DPG to the utility grid, which might cause unwanted protection tripping, bad aging of the passive elements or power electronics damage. 
a)

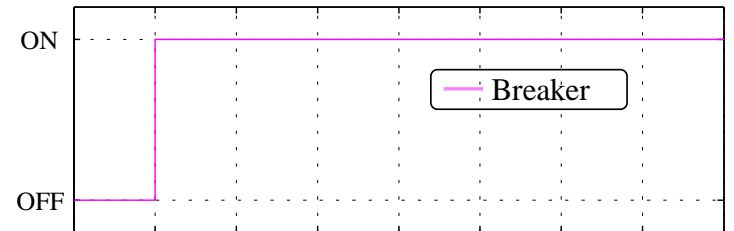

b)

c)
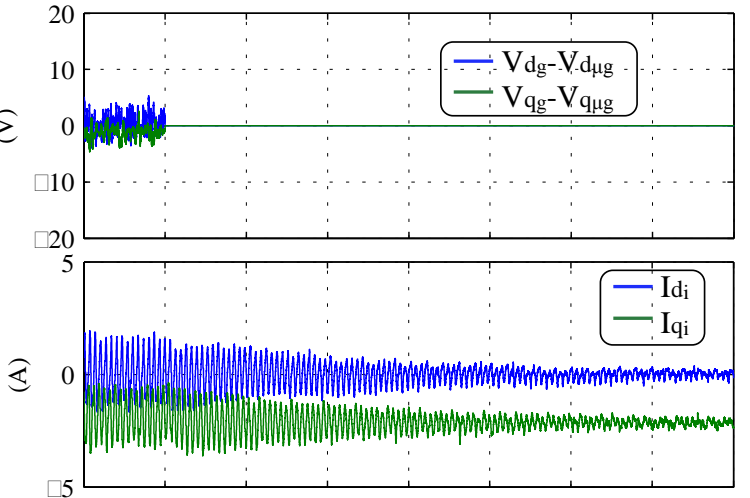

d)

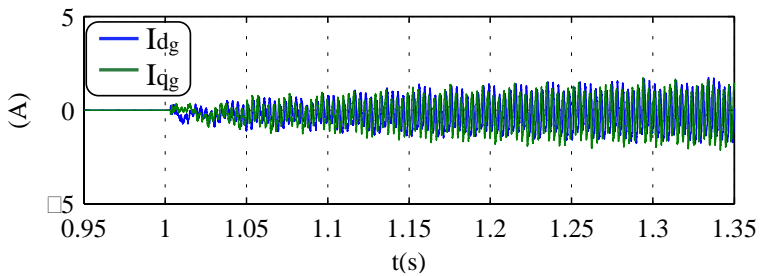

Fig. 17.- Experimental results. Same variables, operating conditions and synchronization method as in Fig. 15, using an LCL filter.

Simulation and experimental results demonstrating the performance of the proposed method have been presented.

\section{REFERENCES}

[1] Z. J. Hong, W. YanTing, W. ZhongJun, Z. Shouzhen, W. Xiaoyu, y S. Xinwei, «Study on microgrid operation modes switching based on eigenvalue analysis», APAP, 1, $445-450,2011$.

[2] C. Blanco, D. Reigosa, F. Briz, J.M. Guerrero, "Synchronization in highly distorted three-phase grids using selective notch filters," IEEE-ECCE, 2641-2648, 15-19 Sept. 2013.

[3] A. Mehrizi-Sani, R. Iravani, "Potential-Function Based Control of a Microgrid in Islanded and Grid-Connected Modes," IEEE Trans. on Pow. Syst., 25 (4), 1883-1891, Nov. 2010.

[4] «Technical Regulation for Electricity generating Facilities of $11 \mathrm{~kW}$ or lower» Energinet Denmark, 2008.

[5] DIN VDE 0126-1-1 «Automatic disconnection device between a generator and the public low-voltage grid», 2006.

[6] ESB Networks «Conditions Governing Connection to the Distribution System: Connections at MV and $38 \mathrm{kV} \&$ Embedded Generators at LV, MV and $38 \mathrm{kV} », 2006$.

[7] «IEEE Guide for Design, Operation, and Integration of Distributed Resource Island Systems with Electric Power Systems», IEEE Std 1547.42011, pp. $1-54$.

[8] M. Liserre, R. Teodorescu, F. Blaabjerg, "Multiple harmonics control for three-phase grid converter systems with the use of PI-RES current controller in a rotating frame," IEEE Trans. on Pow. Electr., 21(3), 836-841, May 2006

[9] R.B. Ridley, "Secondary LC filter analysis and design techniques for current-mode-controlled converters," IEEE Trans. on Pow. Electr., 3(4), 499507, Oct 1988.

[10]J.C.,Vasquez; J.M. Guerrero, M. Savaghebi, J. Eloy-Garcia, R. Teodorescu, "Modeling, Analysis, and Design of Stationary-Reference-Frame Droop-Controlled Parallel Three-Phase Voltage Source Inverters," IEEE Trans. on Ind. Electr., 60 (4) 1271-1280, Apr. 2013. 